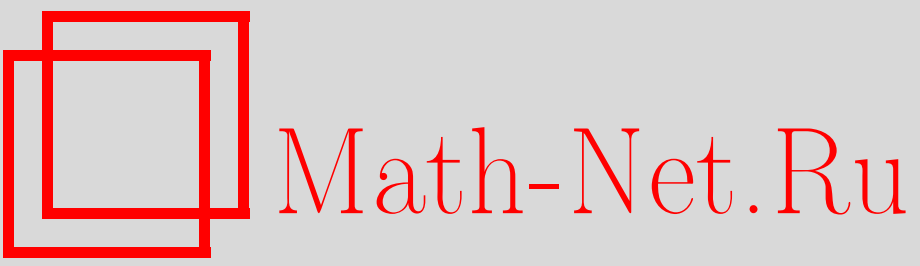

Т. А. Жуковская, Особенности функции минимакса, УМН, 1996, том 51, выпуск 2, 159-160

DOI: https://doi.org/10.4213/rm955

Использование Общероссийского математического портала Math-Net.Ru подразумевает, что вы прочитали и согласны с пользовательским соглашением

http://www.mathnet.ru/rus/agreement

Параметры загрузки:

IP : 54.224 .135 .184

26 апреля 2023 г., 13:44:52 


\title{
ОСОБЕННОСТИ ФУНКЦИИ МИНИМАКСА
}

\author{
Т. А. ЖуковСКАЯ
}

Для уравнений Гамильтона-Якоби с выпуклым по импульсам гамильтонианом слабое решение строится по правилу Максвелла: его значение в каждой точке $q$ пространства-времени задается как наинизшая над $q$ точка соответствующего волнового фронта (см., например, [3]). Типичные особенности этого решения - это особенности множества Максвелла, открытые Томом и изученные Арнольдом и его школой (Л.Н. Брызгалова, В. И. Матов и др.) в 70-х гг. (см. [1]).

М. Шапрон [2] построил слабое решение в общем (не обязательно выпуклом) случае. Используя некоторые конструкции [6], он предложил правило минимакса для выбора нужного значения над каждой точкой пространства-времени.

Число типичных особенностей для минимаксного решения намного больше, чем для функции минимума, дающей решение в выпуклом случае: так, для (ко)размерности 1, 2, 3 существуют, соответственно, 2,7 и 28 различных типов "минимаксных особенностей".

Минимакс производящих семейств. Пусть $\pi_{E}: E \rightarrow B^{m}$ - тривиальное расслоение со слоями $X_{q} \equiv \mathbb{R}^{2 N}, q \in B^{m}$ (в данной работе пространственно-временные переменные не разделяются). Функция $F: E \rightarrow \mathbb{R}$ квадратична на бесконечности (ср. [6]), если существует такая функция $\Theta: E \rightarrow \mathbb{R}$, что каждая $\left.\Theta\right|_{X_{q}}$ является невырожденной квадратичной формой, оба индекса инерции которой равны $N$, причем $F-\Theta$ равномерно $C^{1}$-ограничена в $E$. Далее считаем, что многообразие $B=B^{m}$ компактно и что $\Theta(x, q)=\sum_{i=1}^{N}\left(x_{i}^{+}\right)-\sum_{i=1}^{N}\left(x_{i}^{-}\right)$, где $x_{i}^{+}$и $x_{i}^{-}-$компоненты точки $x=\left(x^{+}, x^{-}\right) \in X_{q} \equiv \mathbb{R}^{N} \oplus \mathbb{R}^{N}$.

Пусть $F: E \rightarrow \mathbb{R}$ - функция, квадратичная на бесконечности. Для $r \in \mathbb{R}$ положим $X_{r, q}=$ $\left\{x \in X_{q}: F(x, q) \leqslant r\right\}$. Тогда (см. [4]) при достаточно больших $r>0$ множество $X_{-r, q}$ (с любым $q$ ) имеет гомотопический тип $N-1$-мерной сферы, в частности, $H_{N}\left(X_{q}, X_{-r, q}\right) \simeq \mathbb{Z}$. Обозначим через $A$ образующую группы $H_{N}\left(X_{q}, X_{-r, q}\right)$, где $r>0$ фиксировано и достаточно велико (так что, в частности, для любого $q \in B$ поле $\operatorname{grad} F(\cdot, q)$ трансверсально границе $\left.X_{-r, q}\right)$. Семейство $F$ определяет функцию минимакса

$$
u_{F}: B \rightarrow \mathbb{R}, \quad u_{F}(q) \equiv \inf _{[\sigma]=A} \max _{x \in|\sigma|} F(x, q)
$$

где $\sigma$ - относительный цикл, реализующий $A$, максимум взят по всем $x \in X_{q}$, принадлежащим носителю $\sigma$, a inf берется по всем реализациям $\sigma$ класса $A$.

Легко доказывается (см. [4]), что для любого $q \in B u_{F}(q)$ - критическое значение функции $F(\cdot, q)$, и что функция $u_{F}: B \rightarrow \mathbb{R}$ липшицева.

Локализация особенностей минимакса общего положения. Пусть $F(x, q)$ - квадратичное на бесконечности семейство общего положения. Тогда для любого $q$ число критических точек $F(\cdot, q)$ конечно. Пусть $q_{0} \in B$ и $x_{1}, \ldots, x_{s}$ - все критические точки $F\left(\cdot, q_{0}\right)$, соответствующие критическому значению $u \equiv u_{F}\left(q_{0}\right)$. Существует такое $\varepsilon>0$, что $\varepsilon$-окрестность $u$ не содержит других критических значений $F\left(\cdot, q_{0}\right)$. Для простоты обозначим $X_{q_{0}} \simeq X, X_{r} \equiv X_{r, q_{0}}$. Для достаточно малых $\varepsilon$ рассмотрим естественные гомоморфизмы

$$
i_{1 *}: H_{N}\left(X, X_{-r}\right) \rightarrow H_{N}\left(X, X_{u-\varepsilon}\right), \quad i_{2 *}: H_{N}\left(X_{u+\varepsilon}, X_{u-\varepsilon}\right) \rightarrow H_{N}\left(X, X_{u-\varepsilon}\right) .
$$

ПреДЛОженИЕ (см. [4]). 1) Образ $i_{1 *}(A)$ чикла $A$ не равен 0 и принадлежит образу гомоморфизма $i_{2 *}$.

2) Пусть $O_{i} \subset X, i=1, \ldots, s,-$ маленькие шары с чентрами в $x_{i}$, тогда для достаточно мальх $\varepsilon>0$

$$
H_{N}\left(X_{u+\varepsilon}, X_{u-\varepsilon}\right) \simeq H_{N}\left(X_{u-\varepsilon} \cup\left(\bigcup_{i=1}^{s} O_{i}\right), X_{u-\varepsilon}\right) \simeq \bigoplus_{i=1}^{s} H_{N}\left(O_{i}, O_{i} \cap X_{u-\varepsilon}\right)
$$


и, более того, аналогичные изоморфизмы имеют место в слое над любой точкой $q \in B$, достаточно близкой $\kappa q_{0}$.

Положим $P \equiv i_{2 *}^{-1}\left(i_{1 *} A\right)$ : это нетривиальньй смежный класс подгруппы $\operatorname{ker}\left(i_{2 *}\right)$. Мы отождествляем $P$ с подмножеством группы $\bigoplus_{i=1}^{s} H_{N}\left(O_{i}, O_{i} \cap X_{u-\varepsilon}\right)$ в соответствии с предыдущим изоморфизмом. Иными словами, циклы $\sigma$, реализующие элементы $P,-$ это наборы $\left(\sigma_{1}, \ldots, \sigma_{s}\right)$, где $\sigma_{i}$ - циклы в $O_{i} /\left(O_{i} \cap X_{u-\varepsilon}\right)$. По определению, вблизи $q_{0}$

$$
u_{F}(q)=\widetilde{u}_{F, P}(q) \equiv \min _{[\sigma] \in P}\left(\max _{i=1, \ldots, s}\left(\max _{x \in O_{i} \cap|\sigma|} F(x, q)\right)\right) .
$$

Основная теОРема. Пусть $F-$ общего положсения. Тогда

(i) При $m \leqslant 5$ функция $u_{F}$ вблизи точки $q_{0} R^{+}$-әквивалентна нормальной форме, принадлежащей некоторому конечному списку (зависящему от $\mathrm{m}$ ); этот список образован ростками функций

$$
\min _{\left(\left[\sigma_{1}\right], \ldots,\left[\sigma_{s}\right] \in P\right)}\left(\max _{i=1, \ldots, s}\left(\max _{x \in\left[\sigma_{i}\right]} F_{\mathcal{N}_{1}, \ldots, \mathcal{N}_{s}}(x, q)\right)\right),
$$

где особенности $\mathscr{N}_{i}$ - из списка простых особенностей [5], [1], сумма $\mu$ их чисел Милнора не превосходит $m+1$ (функиия (1) явно зависит от $\mu-1$ переменных), $\sigma_{i}-$ относительные чиклы, а $P$ обозначает нетривиальный смежный класс по некоторой подгруппе группь $\bigoplus H_{N}\left(\mathcal{N}_{i}\right)_{x_{i}} \equiv \bigoplus_{i=1}^{s} H_{N}\left(O_{i}, O_{i} \cap X_{u-\varepsilon}\right)\left(R^{+}\right.$-muп этой нормальной формы задается набором классов особенностей $\mathscr{N}_{i}$, подгруппой этой группь и ее смежным классом).

(ii) Для всякого $m$ функция минимакса $u_{F}$ локально топологически $R^{+}$-әквивалентна одной из нормальных форм, принадлежсащих некоторому конечному списку (зависящему от $m$ ).

(iii) График функции минимакса $u_{F}$ локально диффеоморфен замкнутому полуалгебраическому множеству, в частности, кусочно гладок.

Я благодарю М. Шапрона за постановку задачи и руководство при ее решении, а также В.А. Васильева, С.М. Гусейн-Заде, В.М. Закалюкина и А.А. Давыдова за полезные обсуждения.

\section{СПИСОК ЛИТЕРАТУРЫ}

[1] Арнольд В. И., Варченко А. Н., Гусейн-Заде С. М. Особенности дифференцируемых отображений. М.: Наука, 1982. [2] Chaperon M. // C. R. A. Sci. Paris. 1991. V. 312. Série I. P. 345-384. [3] Debeneix T. Études de certains systèmes hyperboliques de $N$ equations à $N$ variables, thèse, 1978. [4] Joukovskaja T. Singularités de minimax et solutions faibles d'équations aux derivées partielles, thése de doctorat. Paris, 1994. [5] Thom R. // Bull. AMS. 1960. V. 75. P. 240-284. [6] Viterbo C. // Math. Ann. 1992. V. 292. P. 685-710. 